\title{
OPINIÓN/CARTAS
}

\section{¿Será el metildibromoglutaronitrilo (Euxyl® K400) el alergeno de contacto del año 2005 en Navarra? Will methyldibromo glutaronitrile (Euxyl ${ }^{\circledR}$ K400) be the contact allergen of the year 2005 in Navarra?}

\section{Hervella ${ }^{1,2}$, L. Cascante ${ }^{1}$, J.I. Yanguas ${ }^{2}$, M. Gállego ${ }^{2}$}

El metildibromoglutaronitrilo es uno de los dos ingredientes activos del Euxyl ${ }^{\circledR}$ $\mathrm{K} 400^{1}$ y su principal fuente de sensibilización alérgica. También se conoce con el nombre de 1,2-dibromo-2,4-dicianobutano y es una sustancia antimicrobiana de amplio espectro que se emplea en Europa desde los años 80 como conservante de productos de belleza y sustancias de uso industrial como pinturas de látex, fluidos de corte y adhesivos. Fue diseñado como sustituto de la mezcla metilcloroisotiazolinona/isotiazolinona (Kathon ${ }^{\circledR} \quad$ CG o Euxyl® K100), otro conservante que había demostrado ser una causa frecuente de dermatitis de contacto alérgica ${ }^{2}$. Actualmente su empleo en la fabricación de cosméticos está muy extendido, en parte debido a su eficacia como biocida, pero también debido a que se ha tardado mucho en descubrir su verdadera capacidad sensibilizante ${ }^{2}$.

El metildibromoglutaronitrilo se vende con varios nombres comerciales; los más conocidos son Tektamer 38® en el ámbito industrial y Euxyl® K400 en la industria cosmética $^{1,3-5}$. El Euxyl® K400 se compone en realidad de dos ingredientes activos ${ }^{5,6}$, en proporción 1:4: metildibromoglutaronitrilo y 2-fenoxietanol, aunque este último muy raras veces ha sido el responsable de la sensibilización ${ }^{6-8}$.
En los últimos 10 años la incidencia de alergia de contacto al metildibromoglutaronitrilo ha aumentado progresivamente en Europa y Estados Unidos, y se encuentra ahora en un 2 a $4 \%$ de los pacientes estudiados por sospecha de dermatitis de contacto alérgica ${ }^{9,10}$. En nuestro país los últimos datos epidemiológicos disponibles, documentados por el Grupo Español de Investigación de Dermatitis de Contacto-GEIDC- de la Academia Española de Dermatología y Venereología, en 3.832 pacientes $^{11}$, muestran que este alergeno supuso en 2001 el 1,41\% de las pruebas epicutáneas positivas, casi siempre con relevancia clínica. Desde 2001 el GEIDC, máximo órgano asesor en España sobre sensibilizaciones alérgicas por contacto, recomienda a todos los dermatólogos incluir al Euxyl ${ }^{\circledR}$ K400 en la serie estándar de pruebas epicutáneas $^{12}$

En las consultas de Dermatología del Servicio Navarro de Salud, el Euxyl® K400 se parchea de forma rutinaria en la batería estándar de pruebas epicutáneas desde finales del año 2002. Desde entonces hemos venido observando un alto índice de sensibilizaciones a este conservante. Como ejemplo referimos los datos registrados por uno de los autores (MHG) en los años 2003 y 2004, que agrupan los resultados de 228 enfermos estudiados en

An. Sist. Sanit. Navar. 2005; 28 (1): 105-108

1. Unidad de Dermatología. Hospital García Orcoyen. Estella. Navarra.

2. Servicio de Dermatología. Hospital de Navarra. Pamplona.

Aceptado para su publicación el 8 de febrero de 2005.

\section{Correspondencia}

Marcos Hervella Garcés

Avenida Pío XII, 37-5 B

31008 Pamplona

Tfno: 948273434

E-mail: marcoshervella@telefonica.net 
su consulta por la sospecha de dermatitis de contacto. A todos los pacientes se les realizaron pruebas epicutáneas con la serie estándar del GEIDC, incluyendo al Euxyl® K400 (al 1,5\% en vaselina), más otras series de pruebas específicas en función de la sospecha clínica. El Euxyl ${ }^{\circledR}$ K400 fue el responsable del $2 \%$ de las reacciones positivas en 4 de los 108 enfermos (un $3,7 \%$ ) estudiados en el año 2003 , y del $1,8 \%$ de las pruebas positivas en 5 enfermos de $120(4,2 \%)$ en el 2004. En ocho de los nueve casos las reacciones fueron interpretadas como clínicamente relevantes. Seis pacientes eran mujeres y tres varones. Las manifestaciones clínicas de la alergia a este conservante fueron siempre en forma de dermatitis espongiótica (eccematosa) subaguda o crónica, y de una intensidad leve o moderada. Las lesiones se localizaron con más frecuencia en zonas expuestas-diseminadas (3 enfermos), en dorso de manos-antebrazos (otros 3) y en párpadoscara (2 enfermos). El origen de la sensibilización fue en 6 pacientes el uso de cosméticos -uno de ellos profesional- (Fig. 1), y en los demás se sospechó el contacto con pinturas, en un caso, y con aceites de maquinaria o productos agrícolas, en otro.

Nos parece oportuno comentar que una de las pacientes, mujer de 43 años, que padecía placas eritematosas en párpados superiores desde hacía un año, y que ya había sido estudiada previamente en otro centro con la serie estándar antigua de pruebas epicutáneas, con pruebas negativas y diagnosticada de una dermatitis seborreica, resultó ser alérgica al metildibromoglutaronitrilo contenido en una loción limpiadora. Por otro lado, en otro enfermo, varón del que sospechamos una sensibilización por el contacto con diversas grasas y aceites de su trabajo, y que mejoró al evitarlos, nunca pudimos conocer la composición de los productos que manipulaba debido a la deficiente información de las fichas de seguridad de estos aceites, un hecho habitual en los productos químicos de uso industrial, y que sigue desafiando la normativa europea actual y entorpeciendo la labor diagnóstica de los dermatólogos.

La alergia al metildibromoglutaronitrilo está demostrando ser un reto terapéutico

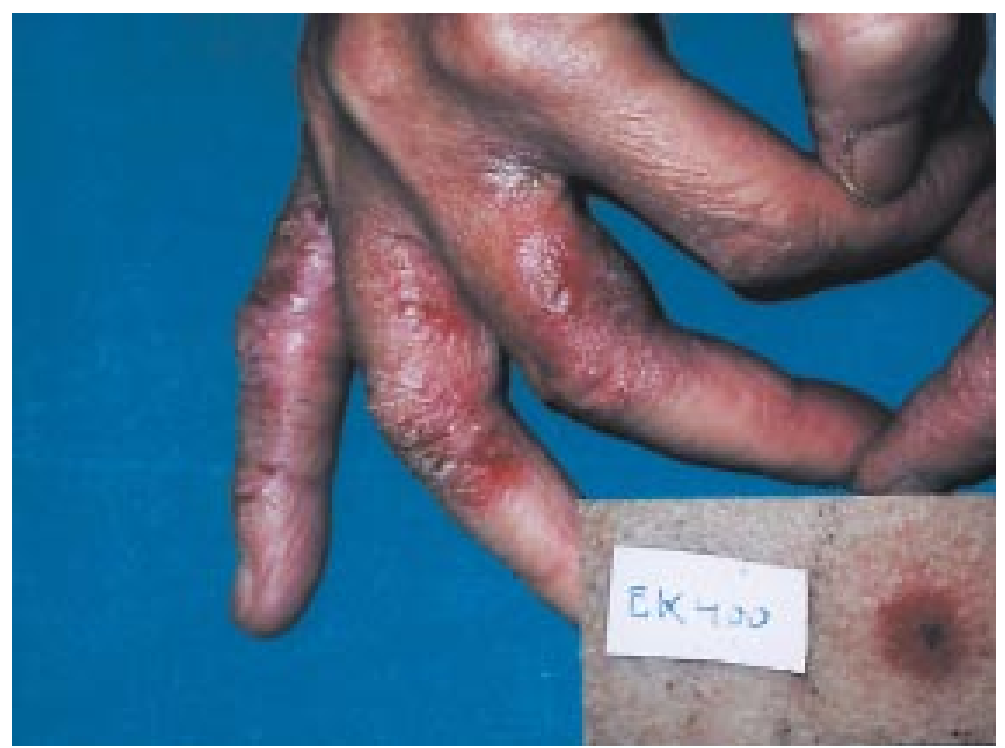

Figura 1. Peluquera de 51 años, que presenta un eccema crónico de manos, con empeoramiento progresivo. Afecta más a la zona de la mano derecha que contacta con el pelo recién lavado de sus clientas y con las espumas fijadoras. Detalle de la prueba epicutánea positiva al Euxyl K400, a las 96 horas. 
en muchos de nuestros enfermos. Una paciente, de profesión peluquera (Fig. 1) con un eccema de manos de larga evolución, no ha mejorado significativamente tras el diagnóstico. Ella regenta un negocio propio e inicialmente manipulaba múltiples cosméticos de una marca muy conocida que contenían metildibromoglutaronitrilo. La paciente ha seguido presentando rebrotes de su dermatitis al utilizar productos de belleza aparentemente exentos de este conservante, después de haber sustituido todos los productos de su peluquería por los de otras marcas. La paciente no es alérgica al otro componente del Euxyl ${ }^{\circledR}$ K400 (fenoxietanol), pero es evidente que algunos de los cosméticos que de forma genérica registran entre sus ingredientes "fenoxietanol" contienen cantidades de metildibromoglutaronitrilo pequeñas pero suficientes como para perpetuar una dermatitis alérgica ${ }^{13}$. Esto puede suponer un verdadero problema para algunos enfermos, dado que el fenoxietanol está presente en casi todos los cosméticos debido a su eficacia contra las pseudomonas.

La sensibilización al Euxyl ${ }^{\circledR}$ K400 es cada vez más frecuente $e^{9-11,14-15}$. En nuestros pacientes fue el segundo sensibilizante más frecuente entre los diagnosticados de alergia a cosméticos, después de las fragancias, y fue un alergeno tan común como la parafenilendiamina o el tiuram. El Euxyl ${ }^{\circledR}$ K400 también fue causa de una dermatosis profesional en al menos uno de nuestros pacientes, y sospechamos su implicación en otros casos del ámbito laboral.

Aunque no disponemos aún de un registro completo que integre los datos de todos los enfermos estudiados en las consultas de dermatología, estimamos que este año se podrían detectar entre 20 y 30 casos de alergia al Euxyl® K400 en Navarra, y nos preguntamos cuántos enfermos (posiblemente otros tantos o más) quedarán sin diagnosticar por no haberse sometido a las pruebas o por no haber sido estudiados según las recomendaciones de la Academia Española de Dermatología y Venereología. En todo caso, parece claro que este conservante será el alergeno de contacto del año 2005 en nuestra comunidad.

\section{BIBLIOGRAFÍA}

1. Schulke \& Mayr GmbH Company. Euxyl® K400. Product information. NorderstedtGerman 1988.

2. Bruze M, GRUVBerger B, Agrup G. Sensitization studies in the guinea pig with the active ingredients of Euxyl® K400. Contact Dermatitis 1988; 18: 37-39.

3. Mathias CGT. Contact dermatitis to a new biocide (Tektamer 38) used in a plastic glue formulation. Contact Dermatitis 1983; 9: 418.

4. Tosti A, Guerra L, Bardazzi F, Gasparri F. Euxyl® K400: a new sensitizer in cosmetics. Contact Dermatitis 1991; 25: 89-93.

5. De Groot AC, van Ginkel CJ, WeiJland JW. Methyldibromo-glutaronitrile $\quad$ Euxyl ${ }^{\circledR}$ $\mathrm{K} 400$ ): an important "new" allergen in cosmetics. J Am Acad Dermatol 1996; 35: 743-747.

6. HAUSEN BM. The sensitizing potency of Euxyl ${ }^{\circledR}$ K400 and its components 1,2dibromo-2,4-dicyanobutane and 2phenoxyethanol. Contact Dermatitis 1993; 28: 149-154.

7. Lovell CR, White IR, Boyle J. Contact dermatitis from phenoxyethanol in aquous cream BP. Contact Dermatitis 1984; 11: 187.

8. Hernández B, Ortiz de Frutos FJ, García M, Palencia S, García MC, Iglesias L. Contact urticaria from 2-phenoxyethanol. Contact Dermatitis 2002; 47: 54.

9. Wilkinson JD, ShaW S, Andersen KE, BRandao FM, BRUYNZEEL DP, BRUZE M et al. Monitoring levels of preservative sensitivity in Europe. A 10-year overview (1991-2000). Contact Dermatitis 2002; 46: 207-210.

10. MARKS JG JR, Belsito DV, DeLeo VA, Fowler JF JR, Fransway AF, Maibach HI et al. North American Contact Dermatitis Group patch tests results, 1996-1998. Arch Dermatol 2000; 136: $272-273$.

11. García-Bravo B, Conde-Salazar L, De la Cuadra J, FERNÁNDEZ-REDONDO V, FERNÁNDEZ-VOZMEDIANO JM, GuIMARAENS D et al. Estudio epidemiológico de la dermatitis alérgica de contacto en España (2001). Actas Dermosifiliogr 2004; 95 : 14-24.

12. Grupo Español de Investigación de Dermatitis de Contacto (GEIDC), de la Academia Española de Dermatología y Venereología. http://www.aedv.es/ gruposdetrabajo/GEIDC. 
13. RASTOGI SC. Analytical control of preservative labelling on skin creams. Contact Dermatitis 2000; 43: 339-343.

14. VAN GINKEL CJ, RUNDERVOORT GJ. Increasing incidence of contact allergy to the new preservative 1,2-dibromo-2,4-dicyanobutane (methyldibromo glutaronitrile). $\mathrm{Br} \quad \mathrm{J}$ Dermatol 1995; 132: 918-920.

15. McFadden JP, Ross JS, Jones AB, Rycroft RJ, SMITH HR, White IR. Increased rate of patch test reactivity to methyldibromo glutaronitrile. Contact Dermatitis 2000; 42: 54-55. 